\title{
REVISIÓN DEL GÉNERO MIMOPHYTUM GREENM. (BORAGINACEAE)
}

\author{
Emmanuel Pérez-Calix ${ }^{1}$ y Alfredo Patiño-Siciliano ${ }^{2}$ \\ ${ }^{1}$ Instituto de Ecología, A.C., Centro Regional del Bajío, Apdo. postal 386, 61600 \\ Pátzcuaro, Michoacán, México. emmanuel.perezcalix@inecol.edu.mx \\ ${ }^{2}$ Instituto Politécnico Nacional, Escuela Nacional de Ciencias Biológicas, \\ Departamento de Botánica, Plan de Ayala y Carpio, 11340 \\ México, D.F., México. apsiciliano@yahoo.com.mx
}

\section{RESUMEN}

Se presenta una descripción morfológica del género Mimophytum Greenm. (Boraginaceae), datos referentes a su distribución geográfica y ecológica y una clave dicotómica para identificar sus elementos. Asimismo, se describen las dos especies conocidas de este grupo vegetal endémico de la Sierra Madre Oriental de México; una de ellas, $M$. benitomartinezii se propone como nueva para la ciencia, pues difiere de $M$. omphalodoides en que lleva el tallo rizomatoso-estolonífero, el haz de la hoja glabro, la lámina foliar con 3 nervios basales, la inflorescencia en forma de cima escorpiodea, ebracteada, los pedicelos más cortos; además, los frutos de la nueva especie tienen los gloquidios dispuestos periféricamente.

Palabras clave: Boraginaceae, México, Mimophytum, taxonomía.

\begin{abstract}
A morphological description of the genus Mimophytum Greenm. (Boraginaceae) is provided, as well as a dichotomous key to the species and ecological and distributional information. The genus is endemic to the Sierra Madre Oriental of Mexico. Morphological descriptions for the two species are provided. One of them, M. benitomartinezii, is proposed as new to science. It differs from $M$. omphalodoides in that the stems are rhizomatousstoloniferous, the upper leaf surfaces are glabrous, the leaf blades possess 3 basal nerves, the inflorescence is an ebracteate, scorpioid cyme, the pedicels are shorter, and the fruits have glochids forming a ring around the edges.
\end{abstract}

Key words: Boraginaceae, Mexico, Mimophytum, taxonomy. 
El género Mimophytum (Boraginaceae) fue propuesto por Jesse M. Greenman (1905) para ubicar a Mimophytum omphalodoides, especie que, de acuerdo con el mencionado botánico, se asemeja a Omphalodes acuminata B. L. Rob. (=O. cardiophylla A. Gray) en el hábito y otros caracteres superficiales, pero a diferencia de ésta y de los demás representantes de Omphalodes su fruto lleva espinas gloquidiadas. Tal rasgo es más común en los miembros de Cynoglossum, pero los mericarpios de Mimophytum son más discoideos y tienen las mencionadas púas únicamente en la porción superior o dispuestas periféricamente.

A un siglo de su propuesta, el género ha permanecido sólo con la especie descrita inicialmente; además, no se han registrado en la literatura colectas adicionales, ni de la zona de donde proviene el ejemplar tipo, ni de ninguna otra localidad. Las actividades encaminadas a la preparación para la flora del Bajío y sus regiones adyacentes resultaron en el hallazgo de una población de Mimophytum omphalodoides en el estado mexicano de Querétaro; además de otra planta que corresponde al mismo grupo y que se propone como especie nueva para la ciencia en la presente colaboración. A la luz de estos hallazgos se presenta una revisión del género.

Mimophytum Greenm., Proc. Amer. Acad. Arts 41: 242. 1905.

Plantas herbáceas perennes, acaulescentes o caulescentes, volubles, rastreras o colgantes; tallos fistulosos, estriados, glabros o esparcidamente pubescentes, o bien en forma de un cáudex; hojas dispuestas de manera alterna o todas basales, sobre peciolos delgados o filiformes, estriados, acanalados, lámina foliar ovada a ovado-lanceolada, ápice agudo, mucronado o acuminado, base redondeada a profundamente cordada, margen entero, membranácea, haz de color verde fuerte, glabro o densamente pubescente a glabrescente, envés pálido, hírtulo, estriguloso, pelos hialinos, cistolíticos, nervios basales 3-7, además de otros 2 suprabasales; inflorescencias 1-3 por planta, ocasionalmente bifurcadas, terminales, cimas escorpioides o racemiformes; pedúnculo delgado, con o sin brácteas, éstas, cuando están presentes, similares a las hojas; flores hermafroditas, actinomorfas, 5-meras, hipóginas; cáliz 5-partido, estrigoso en ambas caras o glabrescente en la interna, persistente, divisiones linear-oblongas, linear-subuladas u oblongo-lanceoladas; corola de color azul, tubular-campanulada, con un tubo corto y 5 ápendices fauciales, limbo 5-lobado, extendido, lóbulos imbricados en la prefloración; estambres 5, adheridos al tubo de la corola, inclusos, anteras pequeñas, obtusas; ovario súpero, 4-lobado, estilo cilíndrico, estigma capitado-comprimido o discoideo; óvulos sub-horizontales; fru- 
to esquizocárpico, mericarpios 4 , algunas veces menos por aborción, deprimidos, aplanados, oblicuamente fijos a la ginobase, provistos de espinas gloquidiadas en la superficie dorsal o en la periferia, superficie ventral y a veces la ventral estrigulosa; semilla 1 por mercarpio, subcircular a piriforme, lisa.

Género de dos especies, endémico de zonas calizas en la porción centro-sur de la Sierra Madre Oriental mexicana. Aunque se conoce únicamente de pocas localidades de los estados de San Luis Potosí, Querétaro y Puebla, consideramos que probablemente habita también en Hidalgo.

Los miembros de Mimophytum se pueden identificar con la siguiente clave:

1. Hojas basales, lámina redondeada a ligeramente cordada en la base, 3-nervada; inflorescencia (incluyendo el pedúnculo) de $20 \mathrm{~cm}$ o menos de largo, ebractea$\mathrm{da}$; flores sobre pedicelos de 2-5(-7) $\mathrm{cm}$ de largo ............. M. benitomartinezii

1. Hojas alternas, lámina cordada en la base, 5-7-nervada; inflorescencia (incluyendo el pedúnculo) de hasta $45 \mathrm{~cm}$ de largo, bracteada; flores sobre pedicelos de $0.4-0.6 \mathrm{~cm}$ de largo M. omphalodoides

Mimophytum benitomartinezii E. Pérez-Calix et A. Patiño-Siciliano sp. nov. TIPO: México. Querétaro: municipio de Jalpan, aprox. $3 \mathrm{~km}$ al $\mathrm{S}$ de Valle Verde, cerro El Pilón. Talud calizo, húmedo y sombreado en ladera con bosque mesófilo de montaña; 1350 m; 16 II 2001, E. Pérez C. y A. Patiño 4117 (holotipo: IEB; isotipos: ENCB, MEXU). Fig. 1.

Planta herbacea perennis, prostrata; folia in rosulis basalibus disposita, petioli (2.5)5-16 cm longi, laminae ovatae vel ovato-lanceolatae, $1.5-8 \mathrm{~cm}$ longae, $1-4.5 \mathrm{~cm}$ latae, apice acutae mucronataeque, basi rotundatae vel leviter cordatae, ad basem 3-nervatae, supra glabrae, subtus hirtulae usque glabrescentes, pilibus cystolithicis; inflorescentia instar cymae scorpioideae, pedunculus ebracteatus, pedicelli capillares, 2-5(-7) cm longi, pilosi; calyx 5-partitus, segmentis lineari-subulatis vel linearioblongis, ca. $2 \mathrm{~mm}$ longis, ca. $0.5 \mathrm{~mm}$ latis; corolla tubulari-campanulata, azurea, tubo ca. $5 \mathrm{~mm}$ longo, limbo 9-11 mm diametro, 5-lobato, lobis oblongis usque orbicularibus, patentibus, 4-5 mm longis, 2.5-3.5 mm latis, appendices fauciales ca. 1 $\mathrm{mm}$ longi; filamenta ca. $0.7 \mathrm{~mm}$ longa; stylus ca. $0.8 \mathrm{~mm}$ longus; mericarpia subcircularia, plana, convexa, ca. $2.5 \mathrm{~mm}$ diametro, ad marginem glochidiato-spinosa, utrinque spinis minutis; semen 1 in quoque mericarpio, subcirculare vel pyriforme, ca. $1 \mathrm{~mm}$ longum et latum, laeve. 


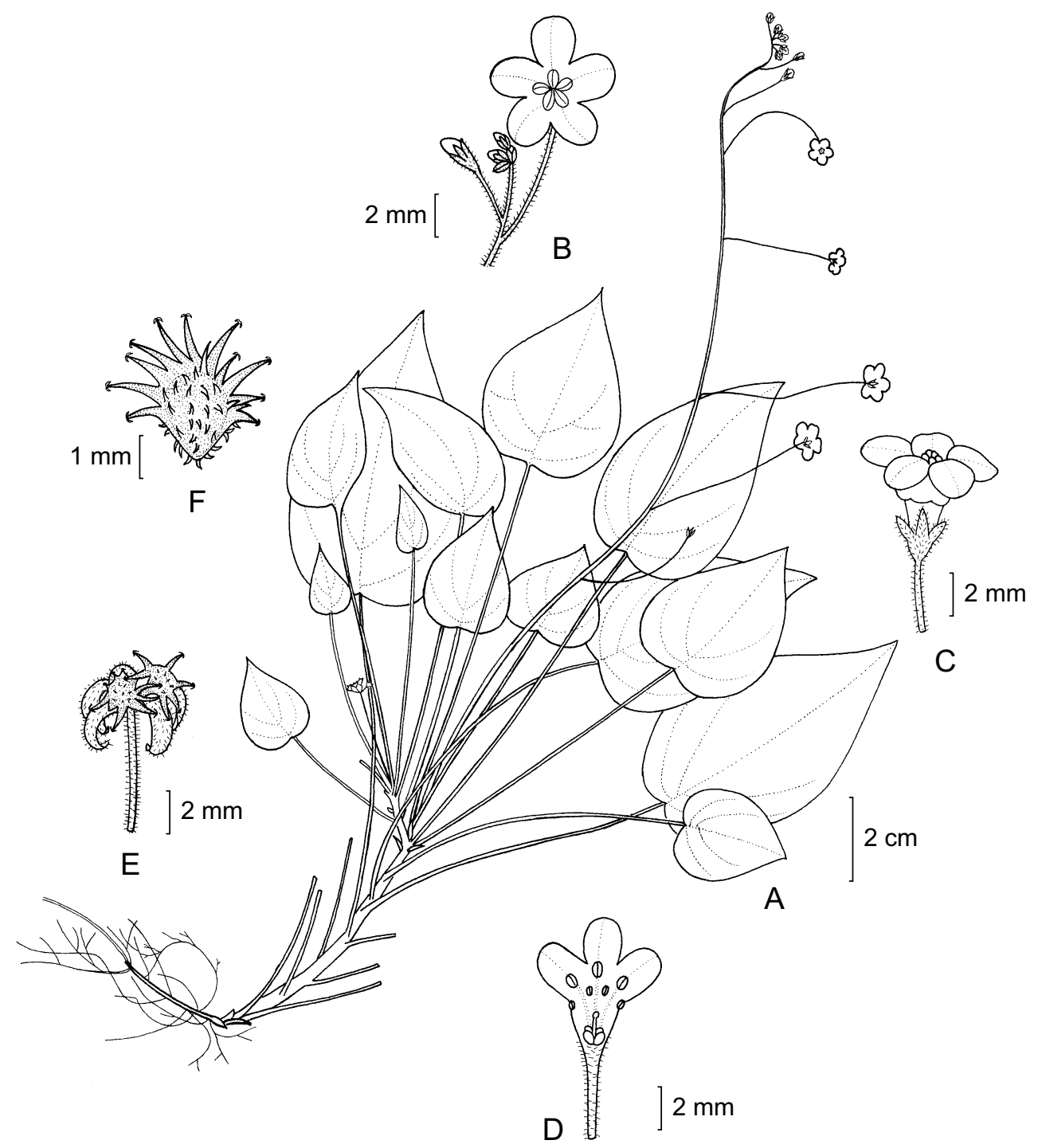

Fig. 1. Mimophytum benitomartinezii E. Pérez-Calix et A. Patiño-Siciliano. A. hábito de la planta con inflorescencia; B. flor en vista superior; C. flor en vista lateral; D. disección de la flor; E. frutos; F. fruto. Ilustración de Rogelio Cárdenas Soriano. 
Planta herbácea perenne, rastrera, de hasta $30 \mathrm{~cm}$ de largo; cáudex estriado, cubierto por restos de los peciolos de otros años de crecimiento, glabro; hojas dispuestas en roseta basal, sobre peciolos delgados, estriados y acanalados, de (2.5-)5$16 \mathrm{~cm}$ de largo, hirsutos a esparcidamente hirsutos, glabrescentes, pelos hialinos, de $1.5 \mathrm{~mm}$ de largo, lámina ovada a ovado-lanceolada, de $1.5-8 \mathrm{~cm}$ de largo, de 1-4.5 cm de ancho, ápice agudo, mucronado, margen entero, densa a esparcidamente ciliado, base redondeada, en ocasiones ligeramente cordada, haz verde fuerte, glabro, envés pálido, hírtulo a glabrescente, pelos hialinos, cistolíticos, caedizos y permaneciendo únicamente la concrescencia cistolítica, largos, lámina con 3 nervios basales y otros 2 suprabasales, resaltados en el envés; inflorescencia 1 por planta, terminal, ocasionalmente bifurcada, en forma de cima escorpioidea, de hasta de 20 $\mathrm{cm}$ de largo; pedúnculo ebracteado, glabro a escasamente piloso, delgado; pedicelo capilar, de 2-5(-7) cm de largo, piloso, pelos hialinos; cáliz profundamente 5-partido, divisiones linear-subuladas o linear-oblongas, de ca. $2 \mathrm{~mm}$ de largo por ca. $0.5 \mathrm{~mm}$ de ancho, estrigosas en la cara externa, glabrescentes en la interna; corola tubularcampanulada, de color azul, de 8-10 $\mathrm{mm}$ de largo, tubo de ca. $5 \mathrm{~mm}$ de largo, ampliándose hacia la parte superior, limbo de 9-11 mm de diámetro, 5-lobado, lóbulos oblongos a subcirculares, extendidos, de 4-5 $\mathrm{mm}$ de largo, de 2.5-3.5 $\mathrm{mm}$ de ancho, bordes enteros, ápice redondeado a truncado, apéndices fauciales presentes, de color café-amarillento, oblongos, planos, con el borde laciniado, papilosos, pruinosos en la cara interna, de ca. $1 \mathrm{~mm}$ de largo por ca. $0.8 \mathrm{~mm}$ de ancho, opuestos a los lóbulos de la corola; estambres inclusos, adnados al tubo de la corola casi $1.5 \mathrm{~mm}$ de la base, filamentos de ca. $0.7 \mathrm{~mm}$ de largo, anteras dorsifijas, triangulares, de $1 \mathrm{~mm}$ de largo y $0.5 \mathrm{~mm}$ de ancho; ovario tetralobado, estilo de $0.8 \mathrm{~mm}$ de largo, 0.4 de ancho, estigma capitado, de $0.3 \mathrm{~mm}$ de diámetro; mericarpios 4 , algunas veces menos por aborción, subcirculares, planos, convexos, de ca. $2.5 \mathrm{~mm}$ de diámetro, gloquidiadoespinosos en la periferia, con espinas diminutas en las dos caras; semilla 1 por mericarpio, subcircular o piriforme, de ca. $1 \mathrm{~mm}$ de largo y ancho, lisa.

La nueva especie florece y fructifica de noviembre a marzo. Habita en el bosque mesófilo de montaña. En altitudes de 990-1350 m.

Paratipos: México: San Luis Potosí: entrando a la gruta en el cerro Silleta, cerca de Xilitla, municipio de Xilitla, L. Paray 480 (MEXU). Querétaro: $3 \mathrm{~km}$ al sureste de Valle Verde, cerro El Pilón, municipio de Jalpan, B. Servín 747 (IEB); cerro El Pilón, ca. $3 \mathrm{~km}$ al S de Valle Verde (La Parada), municipio de Jalpan, E. Pérez 3989 (IEB); ibid., E. Pérez 4464 (IEB); ibid., E. Pérez, A. Patiño y C. Varilla 4445 (IEB). 
A diferencia de la especie descrita por Greenman, Mimophytum benitomartinezii es un planta rastrera, con el tallo rizomatoso-estolonífero; sus hojas están dispuestas en roseta basal, tienen la base redondeada a subcordada, su haz es glabro y son trinervadas basalmente; además, lleva la inflorescencia en forma de cima escorpioidea, ebracteada, los pedicelos más largos, de 2-5(-7) cm, y el fruto detenta las espinas gloquidiadas únicamente en la periferia (Cuadro 1).

Etimología: El epíteto específico de la presente especie se dedica al señor Benito Martínez de la Cruz (Don Benito), quien ha trabajado por alrededor de 20 años con el personal del Centro Regional del Bajío del Instituto de Ecología, A.C. Durante este tiempo ha realizado con gran eficiencia y responsabilidad diversas actividades en el Centro, tales como: montador de ejemplares para el herbario IEB, vigilante de las instalaciones y jardinero.

Cuadro 1. Comparación de caracteres morfológicos de Mimophytum omphalodoides Greenm. y M. benitomartinezii E. Pérez-Calix et A. Patiño-Siciliano.

\begin{tabular}{|c|c|c|}
\hline Carácter & M. benitomartinezii & M. omphalodoides \\
\hline Hábito & herbácea perenne, rastrera & herbácea perenne, colgante a erecta \\
\hline Tallo & cáudex estriado & $\begin{array}{l}\text { fistuloso, estriado, con } \\
\text { ramificaciones dicotómicas }\end{array}$ \\
\hline $\begin{array}{l}\text { Peciolo } \\
\quad \text { largo }(\mathrm{cm}) \\
\text { pubescencia }\end{array}$ & $\begin{array}{l}(2.5-) 5-16 \\
\text { hirsuta a esparcidamente } \\
\text { hirsuta, glabrescente }\end{array}$ & $\begin{array}{l}4-8.5 \\
\text { hirsuta }\end{array}$ \\
\hline $\begin{array}{l}\text { Lámina foliar } \\
\quad \text { forma } \\
\text { largo }(\mathrm{cm}) \\
\text { ancho }(\mathrm{cm}) \\
\text { base } \\
\text { pubescencia } \\
\text { nerviación }\end{array}$ & $\begin{array}{l}\text { ovada a ovado-lanceolada } \\
1.5-8 \\
1-4.5 \\
\text { redondeada a ligeramente } \\
\text { cordada } \\
\text { haz glabro, envés hírtulo a } \\
\text { glabrescente } \\
\text { 3-nervada en la base } \\
\end{array}$ & $\begin{array}{l}\text { ovada } \\
2-7 \\
1-4 \\
\text { profundamente cordada } \\
\text { haz esparcidamente hirsuto, envés } \\
\text { estriguloso } \\
5-7 \text {-nervada en la base }\end{array}$ \\
\hline $\begin{array}{l}\text { Inflorescencia } \\
\text { tipo }\end{array}$ & cima escorpioidea & racimo \\
\hline
\end{tabular}


Cuadro 1. Continuación.

\begin{tabular}{|c|c|c|}
\hline Carácter & M. benitomartinezii & M. omphalodoides \\
\hline $\begin{array}{l}\text { Pedúnculo } \\
\text { pubescencia } \\
\text { brácteas }\end{array}$ & $\begin{array}{l}\text { glabra a escasamente pilosa } \\
\text { ausentes }\end{array}$ & $\begin{array}{l}\text { hirsuto-estrigosa } \\
\text { presentes }\end{array}$ \\
\hline $\begin{array}{l}\text { Pedicelo } \\
\quad \text { largo }(\mathrm{cm}) \\
\text { pubescencia }\end{array}$ & $\begin{array}{l}\text { capilar } \\
2-5(7) \\
\text { pilosa }\end{array}$ & $\begin{array}{l}\text { filiforme } \\
0.4-0.6 \text { en flor, de } 0.4-1.6(2) \text { en } \\
\text { fruto } \\
\text { estrigosa }\end{array}$ \\
\hline $\begin{array}{l}\text { Divisiones del cáliz } \\
\text { largo }(\mathrm{mm}) \\
\text { ancho }(\mathrm{mm}) \\
\text { pubescencia }\end{array}$ & $\begin{array}{l}2 \\
0.5 \\
\text { estrigosa en la cara externa, } \\
\text { glabrescente en la interna }\end{array}$ & \begin{tabular}{|l}
$2.5-3$ \\
1 \\
estrigosa
\end{tabular} \\
\hline $\begin{array}{l}\text { Corola } \\
\text { tubo } \\
\text { largo }(\mathrm{mm}) \\
\text { lóbulos } \\
\text { forma } \\
\text { largo }(\mathrm{mm}) \\
\text { ancho }(\mathrm{mm}) \\
\end{array}$ & $\begin{array}{l}\text { ca. } 5 \\
\text { oblonga a subcircular } \\
4-5 \\
2.5-3.5\end{array}$ & \begin{tabular}{|l|}
$2-3.5$ \\
\\
oblonga \\
$2-3$ \\
$2-3.5$ \\
\end{tabular} \\
\hline $\begin{array}{l}\text { Fruto } \\
\text { ornamentación } \\
\text { diámetro }(\mathrm{mm})\end{array}$ & $\begin{array}{l}\text { gloquidiado-espinoso en } \\
\text { la periferia, con espinas } \\
\text { diminutas en las dos caras } \\
\text { ca. } 2.5\end{array}$ & $\begin{array}{l}\text { gloquidiado-espinoso sobre el } \\
\text { dorso, espinoso en el vientre, } \\
\text { diminutamente estriguloso en } \\
\text { ambas superficies } \\
\text { ca. } 3\end{array}$ \\
\hline
\end{tabular}

Mimophytum omphalodoides Greenm., Proc. Amer. Acad. Arts 41: 242-243. 1905. Tipo: México, Puebla, Estación Honey, C. G. Pringle 8822 (holotipo GH, isotipos en MEXU).

Planta herbácea perenne, colgante-decumbente a erecta, de $60 \mathrm{~cm}$ o más de largo; tallos fistulosos, estriados, con ramificaciones bifurcadas, piloso-hirsutos; hojas alternas, peciolos delgados, de 4 a $8.5 \mathrm{~cm}$ de largo, hirsuto, lámina ovada, de 2-7 $\mathrm{cm}$ de largo, de $1 \mathrm{a} 4 \mathrm{~cm}$ de ancho, ápice agudo a acuminado, margen entero, densa a esparcidamente ciliado, base profundamente cordada, haz verde fuerte, esparcidamente hirsuto, pelos erectos o adpresos, envés pálido, estriguloso, pelos cistolíticos 
en ambas superficies, lámina delgada y membranácea, con 5-7 nervios evidentes desde la base; inflorescencias 1-3 por planta, terminales, racemiformes, de hasta $45 \mathrm{~cm}$ de largo (incluyendo el pedúnculo); pedúnculo hirsuto-estrigoso, delgado, alargado; brácteas florales sobre peciolos de 0.3-1.8 cm de largo, ovadas, de 0.8-4 $\mathrm{cm}$ de largo por 0.8-3 $\mathrm{cm}$ de ancho, acuminadas, con la base cordada, truncada a redondeada, pubescencia similar a la de las hojas; pedicelos filiformes, de 0.4 a $0.6 \mathrm{~cm}$ de largo en floración, acrescentes en fruto y de 0.4-1.6(-2) $\mathrm{cm}$ de largo, estrigosos, extendidos u ocasionalmente recurvados; cáliz profundamente 5-partido, divisiones oblongo-lanceoladas, de 2.5-3 $\mathrm{mm}$ de largo por ca. $1 \mathrm{~mm}$ de ancho, agudas, estrigosas sobre ambas superficies; corola tubular-campanulada, azul, de 5-6 $\mathrm{mm}$ de largo, tubo de 2-3.5 $\mathrm{mm}$ de largo, ampliándose hacia la parte superior, limbo de $4 \mathrm{~mm}$ de diámetro, 5-lobado, lóbulos oblongos, extendidos, de 2-3 mm de largo por 2-3.5 $\mathrm{mm}$ de ancho, borde irregular, ligeramente ondulado, ápice redondeado, apéndices fauciales presentes, de color amarillento, subesféricos, ligeramente geminados, de $0.5 \mathrm{~mm}$ de diámetro, opuestos a los lóbulos, papilosos, pruinosos en la cara interna; estambres inclusos, adnados al tubo de la corola casi $1 \mathrm{~mm}$ de la base, filamentos de $0.5 \mathrm{~mm}$ de largo, anteras de $1 \mathrm{~mm}$ de largo; ovario tetralobado, estilo de $1 \mathrm{~mm}$ de largo, estigma discoideo; mericarpios 4 o menos, subcirculares, plano-convexos, de ca. $3 \mathrm{~mm}$ de diámetro, gloquidiado-espinosos sobre el dorso, espinosos en el vientre, diminutamente estrigulosos en ambas superficies; semilla 1 por mericarpio, subcircular, de ca. $2 \mathrm{~mm}$ de diámetro, lisa.

Distribución ecológica y fenología: especie calcífila, conocida del centrooriente de México, donde habita en taludes húmedos en el bosque mesófilo de montaña en transición con el bosque tropical subcaducifolio; altitud 900-1500 m. Se encuentra en floración de febrero a mayo.

Material revisado: Querétaro: $2 \mathrm{~km}$ al sureste de Neblinas, municipio de Landa, H. Rubio 1604 (ENCB, IEB, MEXU); ibid., E. Pérez 4502 (IEB). Puebla: Estación Honey, C. G. Pringle 8822 (tipo GH, se consultó la fotografía electrónica de la página WEB de este herbario).

\section{AGRADECIMIENTOS}

Deseamos manifestar nuestro más sincero agradecimiento al Dr. Jerzy Rzedowski por la traducción de la diagnosis de la especie nueva al latín; a él mismo, a la 
maestra Graciela Calderón de Rzedowski y al Dr. Victor Steinman por la cuidadosa revisión del manuscrito preliminar. También manifestamos nuestra gratitud a Rogelio Cárdenas por la ilustración representativa de Mimophytum benitomartinezii. El primer autor agradece el apoyo económico del Instituto de Ecología, A. C. (cuenta 20006), del Consejo Nacional de Ciencia y Tecnología y de la Comisión Nacional para el Conocimiento y Uso de la Biodiversidad.

\section{LITERATURA CITADA}

Greenman, J. M. 1905. Descriptions of spermatophytes from the southwestern United States, Mexico, and Central America. Proc. Amer. Acad. Arts 41: 233-270.

Recibido en octubre de 2008.

Aceptado en marzo de 2009. 